\title{
RFP-based method for real-time tracking of invasive bacteria in a heterogeneous population of cells
}

\author{
Rasaq Olajide Akinsola ${ }^{\mathrm{a}}$, Malik Adewoyin ${ }^{\mathrm{b}}$, Choon-Weng Lee ${ }^{\mathrm{c}}$, Edmund Ui-Hang Sim ${ }^{\mathrm{d}}$, \\ Kumaran Narayanan ${ }^{\text {a,* }}$
}

${ }^{a}$ School of Science, Monash University Malaysia, Bandar Sunway 47500, Selangor Darul Ehsan, Malaysia

${ }^{\mathrm{b}}$ Faculty of Dentistry, Universiti Kebangsaan Malaysia Jalan Muda Abdul Aziz, 50300, Kuala Lumpur, Malaysia

${ }^{\mathrm{c}}$ Institute of Biological Sciences, University of Malaya, 50603, Kuala Lumpur, Malaysia

${ }^{\mathrm{d}}$ Faculty of Resource Science and Technology, Universiti Malaysia Sarawak, 94300, Sarawak, Malaysia

\section{A R T I C L E I N F O}

\section{Keywords:}

Invasion

Quantification

E. coli

Adhesion

Internalization

Imaging flow cytometry

\begin{abstract}
A B S T R A C T
Quantification of bacterial invasion into eukaryotic cells is a prerequisite to unfold the molecular mechanisms of this vector's function to obtain insights for improving its efficiency. Invasion is traditionally quantified by antibiotic protection assays that require dilution plating and counting of colony-forming units rescued from infected cells. However, to differentiate between attached and internalized bacteria vector, this assay requires supplementation by a time-consuming and tedious immunofluorescence staining, making it laborious and reduces its reliability and reproducibility. Here we describe a new red fluorescent protein (RFP)-based highthroughput and inexpensive method for tracking bacterial adherence and internalization through flow cytometry to provide a convenient and real-time quantification of bacterial invasiveness in a heterogeneous population of cells. We invaded MCF-7, A549, and HEK-293 cells with the E. coli vector and measured RFP using imaging flow cytometry. We found high cellular infection of up to $70.47 \%$ in MCF-7 compared to $27.4 \%$ and $26.2 \%$ in A549 and HEK-293 cells, respectively. The quantitative evaluation of internalized E. coli is rapid and celldependent, and it distinctively differentiates between attached and cytosolic bacteria while showing the degree of cellular invasiveness. This imaging flow cytometry approach can be applied broadly to study hostbacteria interaction.
\end{abstract}

\section{Introduction}

Invasive bacteria such as E. coli [1-3], Listeria Monocytogenes [4] and Salmonella typhimurium [5] bind specifically to the integrin receptors for internalization into mammalian cells [6]. Our lab previously adapted E. coli as a DNA delivery vector because this strain has established tools for propagation and modification of DNA constructs using recombineering, which facilitated engineering and subsequent delivery of human genomic BACs, compete with introns, exons, and regulatory elements, into cells [6].

Quantification of bacteria invasion into eukaryotic cells is a necessary prerequisite to unfold the molecular mechanisms of how this vector functions in order to obtain insights to improve its efficiency [7]. These intracellular vectors are traditionally quantified for invasiveness by antibiotics protection assays that require dilution plating and counting of colony-forming units rescued from infected cells after the preferential antibiotic killing of the extracellular bacteria [7]. However, this assay needs to be supplemented by a time-consuming and tedious immunofluorescence staining to differentiate between attached and internalized bacteria vector [8]. Apart from the laborious procedure, this assay lacks reliability and reproducibility as it is prone to error of overestimation and or underestimation of the internalized E. coli vector [9].

Overestimation can occur when viable bacteria that remain attached to the host cell after antibiotics treatment are inadvertently recovered and plated on agar along with the internalized bacteria. On the contrary, underestimation could occur during treatment with a high concentration of antibiotics that translocate into the cells and kill internalized E. coli vector encircled in the endosome [10].

To address these limitations, several rapid and high throughput assays involving direct labelling of bacteria or using an antibodyindependent method have been developed [7,9]. These fluorescent stain methods, however, require the use of complicated staining

\footnotetext{
* Corresponding author. Monash University Malaysia, Bandar Sunway, 47500, Selangor Darul Ehsan, Malaysia.

E-mail address: kumaran.narayanan@monash.edu (K. Narayanan).
} 